\title{
Intracellular persistence of chlamydial major outer- membrane protein, lipopolysaccharide and ribosomal RNA after non-productive infection of human monocytes with Chlamydia trachomatis serovar $\mathrm{K}$
}

\author{
E. SCHMITZ, EVA NETTELNBREKER, H. ZEIDLER*, M. HAMMER, ESTHER MANOR $\dagger$ \\ and J. WOLLENHAUPT
}

Division of Rheumatology, Department of Internal Medicine and Dermatology, Medical School, 3000 Hannover, Germany and †Virology Unit, Faculty of Health Sciences, Ben-Gurion University of the Negev, Beer-Sheva, Israel

\begin{abstract}
Summary. The replication of Chlamydia trachomatis serovar $\mathrm{K}$ was studied in human peripheral blood monocytes (PBMo). The intracellular fate of the bacteria was examined by determining the presence of chlamydial major outer-membrane protein (MOMP), lipopolysaccharide (LPS) and ribosomal RNA (rRNA). In-vitro infection of PBMo with $C$. trachomatis serovar $\mathrm{K}$ was not productive. However, chlamydial MOMP antigen, demonstrated by immunofluorescence, was present in PBMo for up to 14 days. Infected monocytes also contained chlamydial rRNA, measured by in-vitro hybridisation, and LPS, measured by enzyme immunoassay, for up to 14 days. These data are compatible with the hypothesis that the infection of PBMo with $C$. trachomatis may play a role in the systemic distribution of chlamydial antigens, leading to systemic manifestations of urogenital chlamydial infection.
\end{abstract}

\section{Introduction}

Chlamydia trachomatis is an obligately intracellular bacterium whose various serotypes cause several ocular, pulmonary and genital diseases in man. ${ }^{1,2} \mathrm{~A}$ common feature of chlamydial infections is their tendency to persist if not adequately treated. ${ }^{3} C$. trachomatis biovar II (serovars $\mathrm{L}_{1}-\mathrm{L}_{3}$ ) causes lymphogranuloma venereum ( $\mathrm{LGV}$ ), characterised by penetration of the epithelial barrier and infection of inguinal lymph nodes with subsequent ulceration. ${ }^{1,2}$ In contrast, infection with $C$. trachomatis biovar I (serovars $\mathrm{A}-\mathrm{K}$ ) tends to be locally restricted to epithelial mucosal cells and is generally thought to be characterised by its lack of invasiveness. ${ }^{4-6}$ Recently, however, chlamydial antigen has been demonstrated intra-articularly in the inflamed joints of patients with Reiter's syndrome or reactive arthritis after urogenital infection with $C$. trachomatis serovars D-K. ${ }^{7-9}$ The intra-articular chlamydial antigen was observed primarily in synovial macrophages. ${ }^{9}$

Therefore, mononuclear phagocytes may play a key role in the systemic distribution and extra-urogenital persistence of C. trachomatis in chlamydial diseases in man. ${ }^{10,11}$ Nevertheless, the mechanisms of chlamydial antigen persistence in mononuclear cells are poorly understood. In this study we investigated the rep-

Received 22 June 1992; revised version accepted 15 Sept. 1992.

* Correspondence should be sent to Professor H. Zeidler. lication of $C$. trachomatis serovar $\mathrm{K}$ in human monocytes and the fate of chlamydial components.

\section{Materials and methods}

\section{Preparation of infectious elementary bodies $(E B)$}

Infectious $\mathrm{EB}$ of $C$. trachomatis serovar $\mathrm{K}$ (UW/31/Cx; Washington Research Foundation, Seattle, USA) were grown in HEp-2 cells (human larynx carcinoma epithelial line) in RPMI 1640 medium, supplemented with fetal calf serum (Biochrome, Berlin, Germany) $10 \% \mathrm{v} / \mathrm{v}$, L-glutamine $1 \%$ $\mathrm{w} / \mathrm{v}$, vancomycin $0.1 \% \mathrm{w} / \mathrm{v}$, gentamicin $0.1 \% \mathrm{w} / \mathrm{v}$, i.e., "growth medium", and containing glucose $1 \%$ $\mathrm{w} / \mathrm{v}$ and cycloheximide $1 \mu \mathrm{g} / \mathrm{ml}$, i.e., "infection medium" (all chemicals were from Sigma). Cultures were incubated for $48 \mathrm{~h}$ at $37^{\circ} \mathrm{C}$ in an atmosphere of $\mathrm{CO}_{2} 5 \% \mathrm{w} / \mathrm{v}$. The organisms were purified in a discontinuous gradient of Urografin (Schering, Berlin, Germany) by ultracentrifugation as described by Caldwell et al. ${ }^{12}$ Purified EB were resuspended in sucrose phosphate buffer $(0.01 \mathrm{M}$ sodium phosphate, $0.25 \mathrm{M}$ sucrose, $5 \mathrm{~mm}$ L-glutamic acid, $\mathrm{pH} 7 \cdot 2$ ), frozen in $0 \cdot 1-\mathrm{ml}$ volumes and stored at $-80^{\circ} \mathrm{C}$.

\section{Determination of chlamydial infectivity}

The infectivity of purified EB or infected monocytes 
was determined by titration on HEp-2 cells. Infected mononuclear phagocytes were treated to release bacteria by thawing the frozen samples in an ice-bath over a 15 -min period followed by sonification for $2 \mathrm{~min}$. Suspensions containing HEp-2 cells $3 \times 10^{5} / \mathrm{ml}$ in growth medium were seeded as $0.1 \mathrm{ml} /$ well in 96well microtitration plates (Nunc, Wiesbaden, Germany) and grown for 2 days at $37^{\circ} \mathrm{C}$ in an atmosphere of $\mathrm{CO}_{2} 5 \% \mathrm{v} / \mathrm{v}$ to confluent monolayers. Ten-fold dilutions of infective samples in infection medium were added as $0.05 \mathrm{ml} /$ well and the cultures were incubated for $48-60 \mathrm{~h}$ at $37^{\circ} \mathrm{C}$. For detection of typical large inclusion bodies by light microscopy, the cells were fixed with absolute ethanol and an indirect immunoperoxidase assay ${ }^{13}$ was performed as follows. Cells were first incubated for $1 \mathrm{~h}$ with the serum of a C. trachomatis antibody-positive patient with a specific IgG titre of $>256$ (Ipazyme test, Medac, Hamburg, Germany) and further incubated with a peroxidaseconjugated goat-anti-human IgG antibody (Sigma) diluted 1 in 40 in PBS. After addition of the substrate 4-chloro-1-naphthol (Savyon, Beer-Sheva, Israel), 1 in 2 in PBS, inclusion bodies were seen as black dots which could be easily identified and counted by light microscopy. The numbers of inclusion bodies/volume were expressed as inclusion forming units (IFU) $/ \mathrm{ml}$.

\section{Purification and chlamydial infection of human peripheral blood monocytes (PBMo)}

Human monocytes were prepared by adherence to plastic. Mononuclear cells from peripheral blood of healthy donors were prepared by Ficoll-Hypaque density gradient centrifugation by the method of Böyum. ${ }^{14}$ The cells, $(5-6) \times 10^{6} / \mathrm{ml}$ in growth medium, were added in 2-ml volumes to six-well cell-culture plates or in 4-ml volumes to 50-ml tissue-culture flasks (Greiner, Nördlingen, Germany) and incubated at $37^{\circ} \mathrm{C}$ in an atmosphere of $\mathrm{CO}_{2} 5 \% \mathrm{v} / \mathrm{v}$. After $2 \mathrm{~h}$, nonadherent cells were removed by vigorous washing in Hanks's Balanced Salts Solution (HBSS, Sigma). About $10-15 \%$ of the inoculated mononuclear cells remained as adherent cells, of which $80-85 \%$ were $C D$ $11 \mathrm{~b}$ antigen-positive or non-specific esterase-positive. ${ }^{15}$ Such cells were considered to be purified PBMo.

Infection of PBMo was performed in the original wells. A separate culture well was used for counting the total number of $\mathrm{PBMo} /$ well after plastic adherence and additional wells served as non-infected controls for each day of harvest. Infectious EB were added to the cells at a multiplicity of infection (MOI) of $0.1,0.5$ and $1 \mathrm{IFU} /$ cell, i.e. $(1-2) \times 10^{6} \mathrm{PBMo} /$ well were incubated with $(1-2) \times\left(10^{5}-10^{6}\right) \mathrm{EB}$ in growth medium at $37^{\circ} \mathrm{C}$ in an atmosphere of $\mathrm{CO}_{2} 5 \% \mathrm{v} / \mathrm{v}$. These were the optimal challenge doses; a higher MOI resulted in a cytotoxic effect for the PBMo. In each experiment, $3 \times 10^{5}$ adherent HEp-2 cells/well in a 24-well microtitration plate were infected at a MOI of 1 , as a control.
Unabsorbed chlamydiae were removed $2 \mathrm{~h}$ postinfection (p.i.) by washing five times in HBSS. Fresh growth medium was added to the cells. A control microscopic examination was performed daily and the viability of PBMo was checked by frequent trypan blue dye-exclusion tests. At least $80 \%$ of the PBMo were alive on day 14 . The cells were harvested at intervals $(0,1,7$ and 14 days p.i.) by scraping carefully with a rubber policeman. Samples were stored at $-70^{\circ} \mathrm{C}$.

To exclude any interference of plastic-adherent extracellular chlamydia with the numbers of infective particles inside PBMo, a control experiment was performed. PBMo were first cultured and infected in 250 -ml culture flasks. After incubation for $2 \mathrm{~h} \mathrm{at} 37^{\circ} \mathrm{C}$, infected adherent PBMo were washed five times with HBSS, but then scraped carefully from the plastic base of the culture flask. The PBMo suspensions were washed three times with HBSS and then (1-2) $\times 10^{6}$ cells $/ \mathrm{ml}$ were seeded in 2- $\mathrm{ml}$ volumes in a six-well plate. At days 0, 1, 7 and 14, re-adherent cells were harvested and washed three times with HBSS.

\section{Immunofluorescence microscopy of chlamydial particles}

Chlamydial major outer membrane protein MOMP was detected by a direct immunofluorescence technique. ${ }^{16}$ After harvesting infected PBMo, cytocentrifuge smears, each containing $c .5 \times 10^{4}$ cells, were prepared (Cytospin, Shandon). They were fixed for 10 min with methanol $100 \%$ and were further incubated with a fluorescein-conjugated murine monoclonal antibody (MAb; Syva, Palo Alto, CA, USA). This $\mathrm{MAb}$ is directed against a common MOMP epitope of EB and reticulate bodies (RB) of all 15 human serovars of $C$. trachomatis. After incubation for $1 \mathrm{~h}$ in a moist chamber at room temperature, slides were rinsed in distilled water and dried in air. A drop of mounting fluid (PBS, $10 \% \mathrm{v} / \mathrm{v}$, glycerol, $90 \% \mathrm{v} / \mathrm{v}$ ) was applied and a coverslip was added. All samples were screened with an epifluorescence microscope (Leitz, Wetzlar, Germany). Only brightly shining green particles identified in PBMo were counted as fluorescent particles (f.p.). The sensitivity of the method was determined with serial dilutions of purified $\mathrm{EB}$ and the detection limit was $10 \mathrm{IFU} / \mathrm{ml}$.

\section{Determination of chlamydial lipopolysaccharide (LPS)}

The presence of chlamydial LPS in infected PBMo was determined by an enzyme immunoassay (IDEIA ${ }^{\circledR}$ Chlamydia test, Novo BioLabs, Cambridge) with genus-specific Fab-fragments of a MAb against chlamydial LPS and an alkaline-phosphatase substrate with an alcohol-oxidoreductase-diaphorase amplification system. PBMo were harvested and suspended in $1 \mathrm{ml}$ of EIA transport medium (IDEIA Chlamydia test). The determination of chlamydial LPS was 


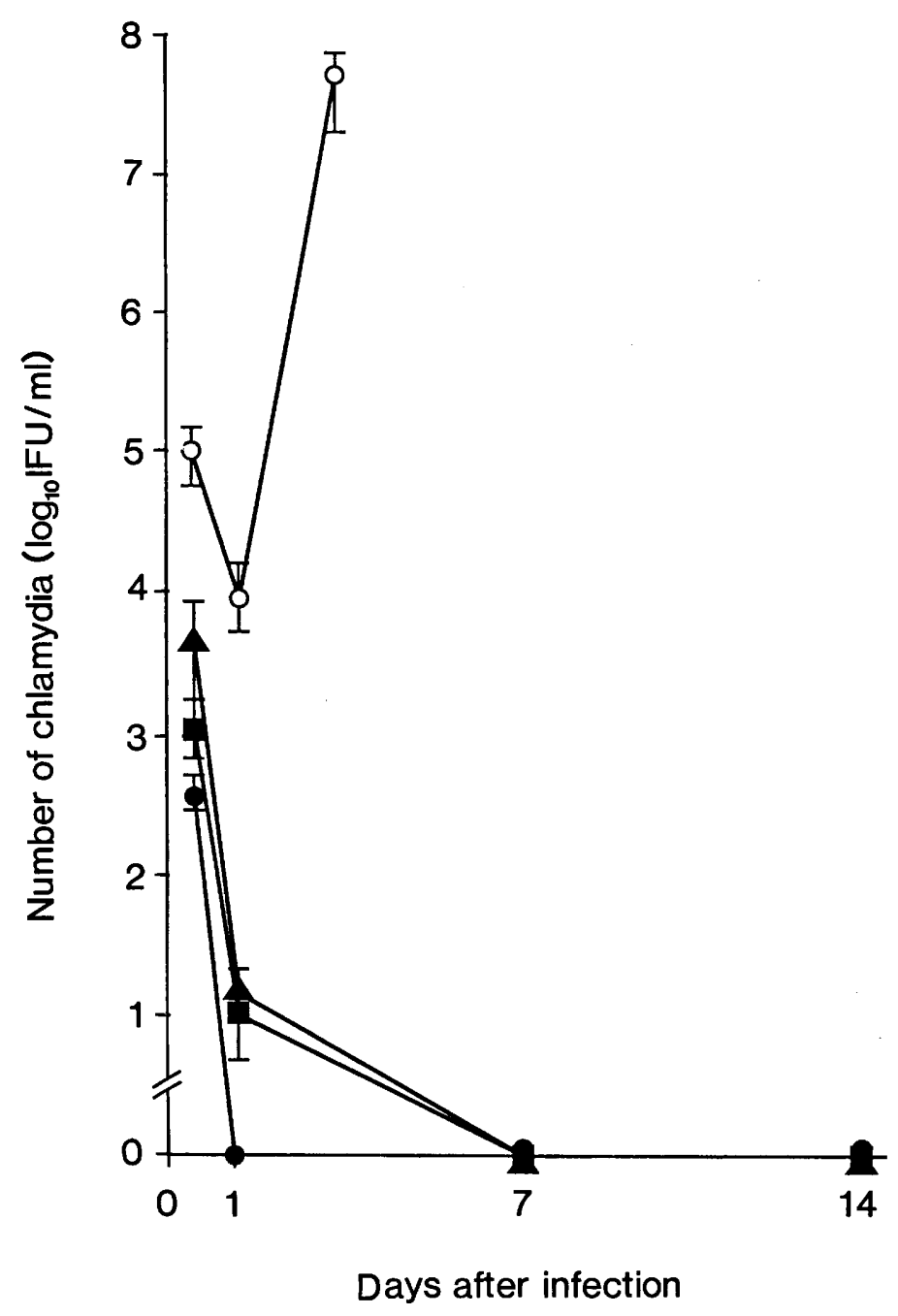

Fig. 1. Infectivity of $C$. trachomatis. One-step growth curves of chlamydiae after infection of PBMo with C. trachomatis at MOI 1 ( $\mathbf{\Delta}$; $\mathrm{n}=6), 0.5(\mathbf{\square} ; \mathrm{n}=3)$ and $0.1(\mathbf{O} ; \mathrm{n}=4)$. The numbers of chlamydiae were determined by titration on HEp- 2 cells and expressed as IFU $/ \mathrm{ml}$. In control experiments $(\mathrm{n}=4)$, growth in HEp-2 cells infected with $C$. trachomatis at MOI $1(O)$ was determined. Results are indicated as means, bars represent $\mathrm{SD}$.

performed according to the manufacturer's instructions. Relative concentrations of LPS in infected monocytes are expressed as extinction at $492 \mathrm{~nm}$. The cut-off for each test procedure was calculated as the mean of three negative controls plus $0 \cdot 2$. The sensitivity of the EIA to detect chlamydial LPS in cell cultures, as evaluated by a serial dilution analysis of EB suspended in transport medium, was $100 \mathrm{IFU} / \mathrm{ml}$.

\section{Determination of chlamydial ribosomal RNA}

Chlamydial rRNA was measured by in-vitro hybridisation with a single-stranded chemiluminescentlabelled DNA probe. The commercially available DNA probe (PACE 2, Gene-probe, San Diego, USA) recognises all 15 human serovars of $C$. trachomatis and reacts specifically with complementary rRNA sequences of $C$. trachomatis. After harvesting mononuclear cells, samples containing $(3-6) \times 10^{5}$ cells were transferred to $1.5-\mathrm{ml}$ reaction tubes and centrifuged at $30000 \mathrm{~g}$ (Biofuge, Heraeus, Hamburg, Germany). The pellets were resuspended in $100-\mu \mathrm{l}$ volumes of lysis buffer (Gene-probe) and incubated for at least 3 days. For the test procedure, samples were transferred to test tubes and $100 \mu 1$ of the acridinium ester-labelled probe reagent was added to each specimen. After a differential hydrolysis to inactivate the non-hybridised DNA, light emission from the DNA-RNA hybrid was measured in a luminometer (Leader I ${ }^{\infty}$, Biermann, Bad Nauheim, Germany). Concentrations of rRNA are expressed as relative light units. Three negative and one positive control were included in each test kit. Non-infected 


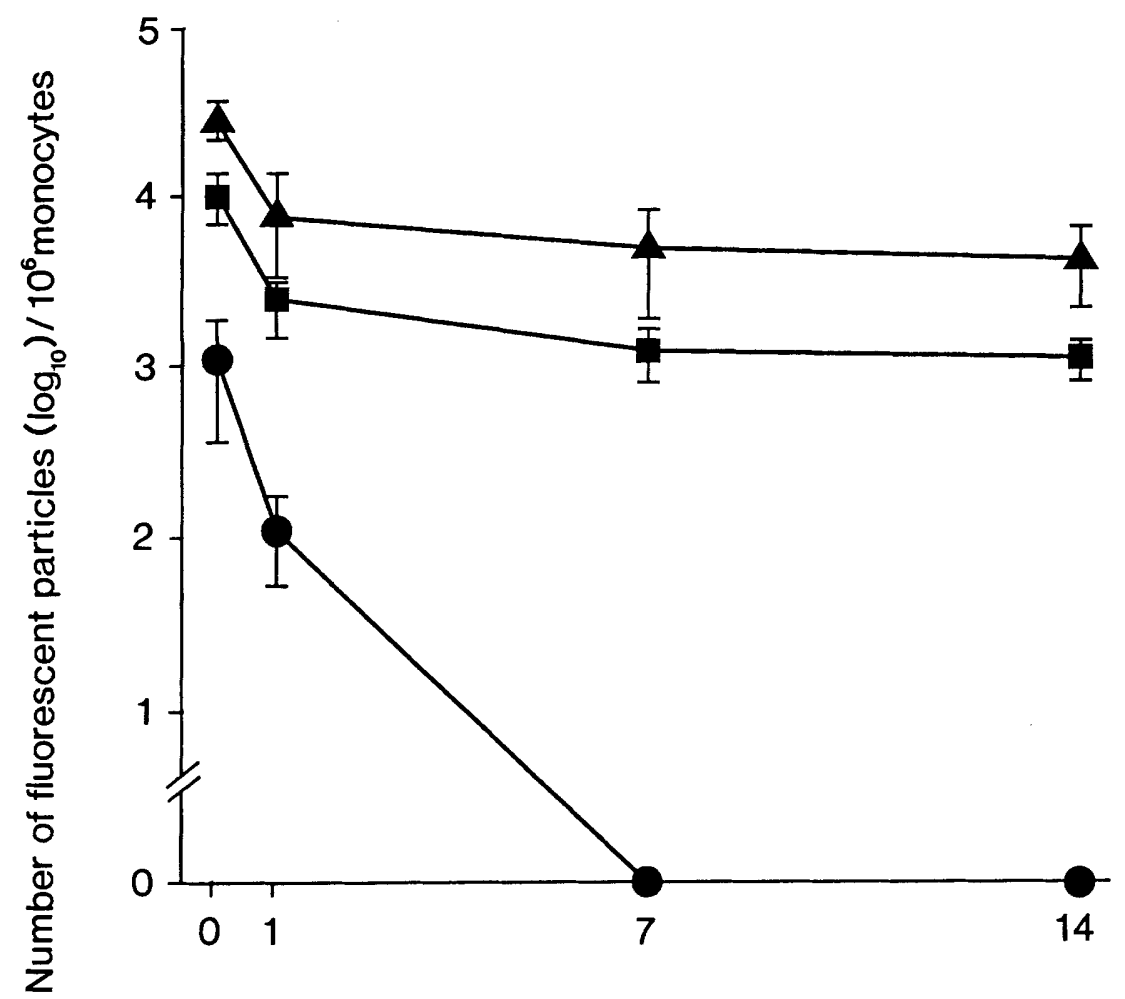

Days after infection

Fig. 2. Determination of chlamydial MOMP in infected PBMo by direct immunofluorescence in PBMo infected with C. trachomatis at MOI $1(\boldsymbol{\Delta} ; \mathrm{n}=6), 0.5(\boldsymbol{\square} ; \mathrm{n}=3)$, and $0.1(\bullet ; \mathrm{n}=4)$. Results are indicated as means, bars represent SD.

PBMo were used as additional negative controls. According to our experience, the test has a detection limit for chlamydial rRNA of at least $50 \mathrm{IFU} / \mathrm{ml}^{17}$

\section{Results}

To study the fate of $C$. trachomatis in PBMo the presence of specific MOMP, LPS and rRNA was investigated over a period of 14 days after infection.

\section{Infectivity of $C$. trachomatis in PBMo cultures}

PBMo could not be infected productively with $C$. trachomatis serovar $\mathrm{K}$. The one-step growth curves of C. trachomatis in PBMo at MOI $0.1,0.5$ and 1 are illustrated in fig. 1. After the removal of unabsorbed bacteria at $2 \mathrm{~h}$ p.i. (i.e. day 0 ), $3-5 \%$ of the initial inoculum could be detected by titration on HEp-2 cells. The number of infectious particles further decreased during the first day. After day 1, with an MOI of 0.5 or 1 , inclusions were scarcely detectable; no IFU were found at MOI $0 \cdot 1$. In none of the experiments was a productive infection observed on day 7 or 14 p.i. In contrast, infection of HEp-2 cells with C. trachomatis at MOI 1 was productive. After an initial decline in the number of infective particles after 1 day, indicating the transformation of infectious EB into non-infectious RB in the life-cycle of chlamydia, the number of infective particles had reached a high level by day 4 . Typical inclusion bodies were visible 1-2 days p.i., indicating a productive infection with the formation of new infectious EB.

Thus, C. trachomatis did not infect PBMo productively, in contrast to its ability to replicate in HEp- 2 cells. The experiments to exclude the possible influence of plastic-adherent extracellular $C$. trachomatis on the infectivity for PBMo demonstrated no alteration in these kinetics.

\section{Detection of chlamydial MOMP by immunofluorescence}

In contrast to the absence of chlamydial replication, the determination of chlamydial MOMP revealed a considerable number of fluorescent particles (f.p.)/cell at MOI 0.5 and 1 throughout the cultivation period (fig. 2). Mostly single and occasionally multiple round, bright green particles were identified in infected PBMo (fig. 3), compared with only non-specific staining in uninfected PBMo. No clusters of f.p. suspected of being inclusion bodies were found and free 


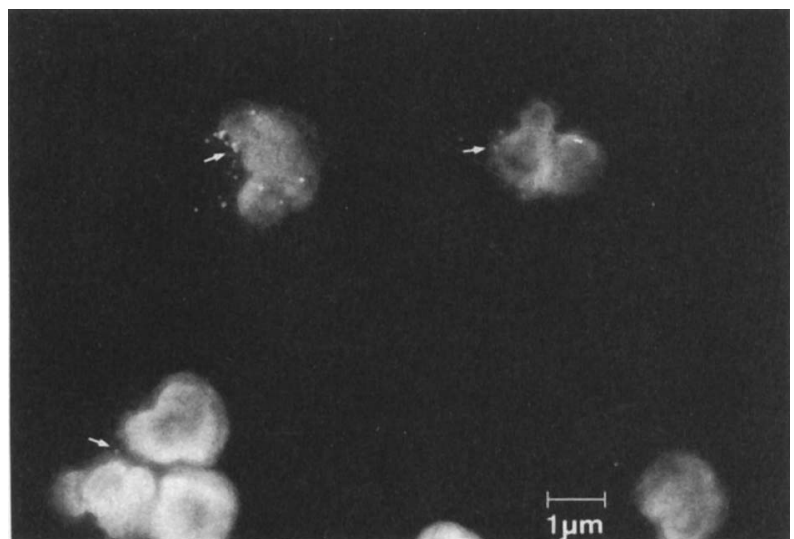

Fig. 3. Immunofluorescence of chlamydial MOMP in infected PBMo at 7 days p.i. Note the single or multiple intracellular fluorescent particles $\rightarrow$

extracellular f.p. could be seen only rarely. The number of f.p. decreased considerably during the first day (paired Student's $t$ test, MOI 1: p $<0.005$; MOI $0.5: \mathrm{p}<0.02$ ) and from days $1-7$ p.i. (MOI $1: \mathrm{p}<$ 0.01 ; MOI 0.5 : n.s.). A constant amount of MOMP was observed from day 7 to day 14 p.i. with MOI 1 and 0.5 . On day 14 , an average of $10 \%$ of the f.p. at day 0 could still be detected in infected PBMo. With MOI $0 \cdot 1$, no persistence of MOMP was observed. Detectable numbers of f.p. could be seen only during the first day p.i. Control experiments, as described above, to exclude any influence of plastic-adherent extracellular EB showed no alterations in these kinetics.

Thus, in infected PBMo, a persistence of MOMP could be observed over the entire culture period of 14 days.

\section{Determination of chlamydial LPS}

The determination of chlamydial LPS in infected PBMo showed dose-dependent concentrations of this cell-wall constituent for up to 14 days p.i. (fig. 4). LPS was identified in PBMo on the first day at all MOI. The concentrations of LPS in PBMo tended to decrease during the cultivation period. At day 14 p.i., however, significant amounts of LPS were still detectable in cells with an MOI of 1 and $0 \cdot 5$, but not at MOI $0 \cdot 1$. No chlamydial LPS was found in non-infected PBMo.

\section{Determination of chlamydial $r R N A$}

To determine whether chlamydial cell-wall components, or chlamydial nucleic acids, or both, persisted in PBMo, the amount of chlamydial rRNA was determined. After infection of PBMo, a significant decline in the concentrations of rRNA was found during the first day p.i. (MOI $1: \mathrm{p}<0.05 ;$ MOI $0.5: \mathrm{p}$ $<0.05$; fig. 5). From day 1 to day 7 a further decrease in chlamydial rRNA concentrations (MOI 1: $\mathrm{p}<$ 0.01 ; MOI $0.5: p<0.01$ ) was demonstrated but the amount of rRNA did not decline further from day 7 to day 14 . Approximately $10 \%$ of the input rRNA concentrations could be detected at 14 days p.i. In contrast, at MOI $0 \cdot 1$, detectable amounts of rRNA could only be found during the first day p.i. No chlamydial rRNA was detected in controls of uninfected PBMo.

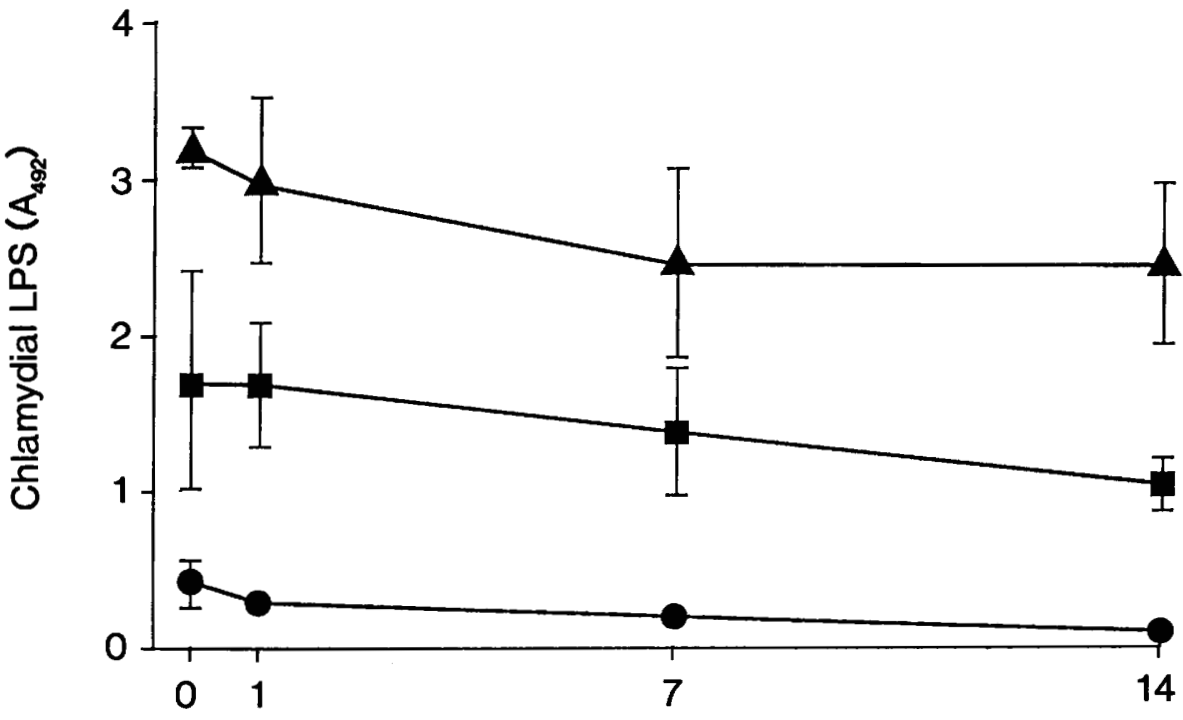

Days after infection 


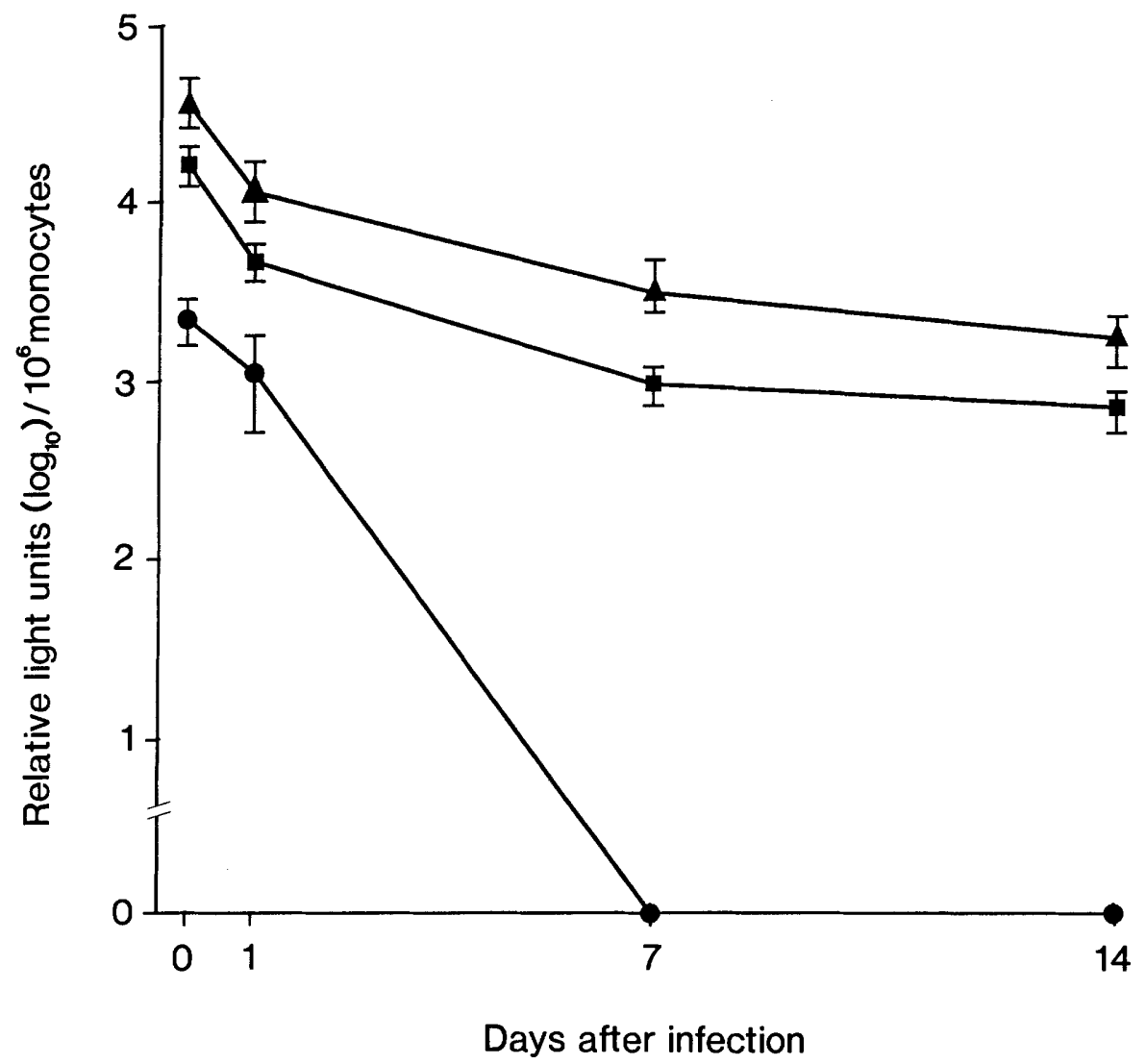

Fig. 5. Determination of kinetics of chlamydial rRNA by hybridisation with a single-stranded DNA probe in PBMo infected with C. trachomatis at MOI $1(\mathbf{\Delta}, \mathrm{n}=6), 0.5(\mathbf{\square} ; \mathrm{n}=3)$ and $0 \cdot 1(\mathbf{\bullet} ; \mathrm{n}=4)$. Results are indicated as means, bars represent SD.

\section{Discussion}

Clinical and in-vitro experimental evidence for persistent infection by $C$. trachomatis has been described frequently. ${ }^{2,3,6,13,18} \mathrm{~A}$ possible involvement of human mononuclear phagocytes in latent and persistent chlamydial infection has been recognised, but the mechanisms of bacterial or antigen persistence are still poorly understood. So far, there have been few studies of the in-vitro interaction between monocytes or macrophages and chlamydia. Limited replication of C. psittaci has been described in human monocytes and their number was reported to increase during the differentiation of the monocytes into macrophages. ${ }^{19,20}$ C. trachomatis serovars $B$ and $\mathrm{L}_{2}$ have been shown to grow to a limited extent in mouse peritoneal macrophages. ${ }^{21}$ With human monocytes and monocyte-derived macrophages ${ }^{18}$ it was found that serovar $L_{2}$ could infect productively in-vitro matured macrophages, while infection in non-differentiated PBMo was non-productive. In a similar study, Bard and Levitt ${ }^{22}$ confirmed these observations, although they found some (i.e. $<0.5 \%$ ) inclusion bodies in monocytes, in contrast to an absolute lack of productive infection described previously. ${ }^{18}$
In contrast to the productive infection of macrophages with the LGV biovar, Yong et al. ${ }^{5}$ demonstrated a non-productive infection with various trachoma biovar I strains (B, C and I). Comparing the fate of $C$. trachomatis serovars $\mathrm{L}_{2}$ and $\mathrm{K}$ in human monocyte-derived macrophages we came to the same conclusions. ${ }^{23}$ Yong et al. ${ }^{5}$ suggested, from their ultrastructural investigations with monocytes cultured for $<1$ week, that both biovars were degraded after phagosome-lysosome fusion. However, the possibility of a persistent or latent infection with the trachoma biovar I in monocytes and macrophages has not been investigated in detail. Therefore, in the present study we examined whether PBMo may be infected persistently by $C$. trachomatis serovar $\mathrm{K}$.

In accordance with previous studies, ${ }^{5,18}$ serovar $\mathrm{K}$, as a member of biovar I, did not infect PBMo productively. The yield of infective particles during the first day p.i. has to be interpreted as the remnant of the initial inoculation. Nevertheless, chlamydial MOMP antigen was identified intracellularly during the entire cultivation period of 14 days. The fluorescent particles in PBMo were mostly single and rarely multiple. As would be expected in the presence of inclusion bodies, intracellular agglomerates could not be detected. The 
observed immunofluorescence staining pattern is consistent with a lack of or insufficient degradation of chlamydial cell walls expressing MOMP antigens. It has long beën known that chlamydial cell wallsespecially those of the EB - are highly resistant to environmental conditions and intracellular degradation. ${ }^{24-26}$ However the persistence of chlamydial envelopes consisting of MOMP and LPS would not explain the present finding of the persistence of the more fragile chlamydial rRNA in PBMo. Owing to the presence of eukaryotic ribonucleases, free rRNA is assumed to be a target for rapid enzymatic digestion. ${ }^{27}$

Our data suggest that infection of human monocytes with $C$. trachomatis serovar $\mathrm{K}$ in vitro leads to an intracellular persistence of complete chlamydial organisms. The findings have several implications for the understanding of systemic manifestations of urogenital infections with $C$. trachomatis. The in-vitro observations may explain recent studies demonstrating the presence of non-infectious particles in samples from joints of patients with Reiter's syndrome, sexually-acquired reactivearthritisand undifferentiated arthritis. ${ }^{7-9}$ Furthermore, the most recent detection of rRNA in synovial membrane biopsies and synovial

\section{References}

1. Schachter J. Chlamydial infections. West J Med 1990; 153: 523-534.

2. Ward ME, Clarke IN. New perspectives in chlamydial biology and development. In: Bowie WR, Caldwell HD, Jones RP et al. (eds) Chlamydial infections. Proceedings of the 7th International Symposium on human chlamydial infections, Harrison Hot Springs. Cambridge, Cambridge University Press. 1990: 3-14.

3. Byrne GI. Interferons, immunity and chlamydiae. In: Byrne GI, Turco $\mathbf{J}$ (eds) Infections and non-viral pathogens. New York, Decker Inc. 1988: 53-73.

4. Pearce JH, Prain CJ. Intracellular association of Chlamydia psittaci with lysosomes during infection of McCoy cells. In: Oriel D, Ridgway G, Schachter J, Taylor-Robinson D, Ward M (eds) Chlamydial infections. Proceedings of the 6th International Symposium on human chlamydial infections, Sanderstead. Cambridge, Cambridge University Press. 1986: 43-46.

5. Yong EC, Chi EY, Kuo C-C. Differential antimicrobial activity of human mononuclear phagocytes against the human biovars of Chlamydia trachomatis. J Immunol 1987; 139 1297-1302.

6. Moulder JW. Comparative biology of intracellular parasitism. Microbiol Rev 1985; 49: 298-337.

7. Ishikawa $\mathrm{H}$, Ohono O, Yamasaki $\mathrm{K}$, Ikuta S, Hirohata $\mathrm{K}$. Arthritis presumably caused by chlamydia in Reiter syndrome. J Bone Joint Surg 1986; 68A: 777-779.

8. Keat A, Dixey J, Sonnex C, Thomas B, Osborn M, TaylorRobinson D. Chlamydia trachomatis and reactive arthritis: the missing link. Lancet 1987; 1: 72-74.

9. Schumacher HR, Magge S, Cherian PV et al. Light and electron microscopic studies on the synovial membrane in Reiter's syndrome. Immunocytochemical identification of chlamydial antigen in patients with early disease. Arthritis Rheum 1988; 31: 937-946.

10. Rothermel CD, Rubin BY, Jaffe EA, Murray HW. Oxygenindependent inhibition of intracellular Chlamydia psittaci growth by human monocytes and interferon- $\gamma$-activated macrophages. J Immunol 1986; 137: 689-692.

11. Sarov I, Geron E, Shemer-Avni Y et al. Implications for persistent chlamydial infections of phagocytemicroorganism interplay. Eur J Clin Microbiol Infect Dis $1991 ; 10: 119-123$. fluid cells from such patients ${ }^{17,28}$ is in full accord with the in-vitro persistence of rRNA in mononuclear phagocytes shown in this study. Although the results obtained in vitro do not necessarily reflect the situation in vivo, they support the hypothesis that monocytes take up $C$. trachomatis in the genital tract and distribute the organism in a Trojan-horse manner through the blood circulation into the joints. Subsequently, the infected PBMo, as precursors of sessile tissue macrophages such as synovial membrane cells, may induce arthritis by production and secretion of pro-inflammatory cytokines, recently described for in-vitro infection of macrophages with C. trachomatis serovar $\mathrm{K} .{ }^{23}$ Further studies are needed to elucidate whether the chlamydiae persist as viable organisms only temporarily arrested in a non-productive or latent state of the growth cycle, or, alternatively, as defective non-viable organisms resistant to degradation by the phagocytic host cell.

We thank Sabine Hopf and Claudia Buntenkötter for superb technical assistance and Drs M. Puls and L. Köhler for their helpful remarks on reading the manuscript. This work was supported by the Deutsche Forschungsgemeinschaft (SFB 244/project C8).

12. Caldwell H, Kromhout J, Schachter J. Purification and partial characterization of the major outer membrane protein of Chlamydia trachomatis. Infect Immun 1981; 31 : 1161-1176.

3. Shemer Y, Sarov I. Inhibition of growth of Chlamydia trachomatis by human gamma interferon. Infect Immun 1985; 48 : 592-596.

14. Böyum A. Isolation of mononuclear cells and granulocytes from human blood. Isolation of mononuclear cells by one centrifugation and sedimentation at $1 \mathrm{~g}$. Scand $J$ Lab Invest 1968; 21 Suppl 97: 77-89.

15. Schmitz E, Ruschen S, Warnatz H. Superoxide anion production by monocytes and synovial fluid macrophages of patients with chronic inflammatory joint disorders. $Z$ Rheumatol 1987; 46: 227-232.

16. Stephens RS, Tam MR, Kuo C-C, Nowinski RC. Monoclonal antibodies to Chlamydia trachomatis: antibody specificities and antigen characterization. $J$ Immunol 1982; 128 1083-1089.

17. Hammer M, Nettelnbreker E, Hopf S, Schmitz E, Pörschke K Zeidler $\mathrm{H}$. Chlamydial rRNA in the joints of patients with chlamydia-induced arthritis and undifferentiated arthritis. Clin Exp Rheumatol 1992; 10: 63-66.

18. Manor E, Sarov I. Fate of Chlamydia trachomatis in human monocytes and monocyte-derived macrophages. Infect Immun 1986; 54: 90-95.

19. Rothermel CD, Rubin BY, Murray HW. $\gamma$-interferon is the factor in lymphokine that activates human macrophages to inhibit intracellular Chlamydia psittaci replication. $J$ Immunol 1983; 131: 2542-2544.

20. Murray HW, Byrne GI, Rothermel CD, Cartelli DM. Lymphokine enhances oxygen-independent activity against intracellular pathogens. $J$ Exp Med 1983; 158: 234-239.

21. Kuo C-C. Cultures of Chlamydia trachomatis in mouse peritoneal macrophages: factors affecting organism growth. Infect Immun 1978; 20 : 439-445.

22. Bard J, Levitt D. Chlamydia trachomatis $\left(\mathrm{L}_{2}\right.$ serovar) binds to distinct subpopulations of human peripheral blood leucocytes. Clin Immunol Immunopathol 1986; 38: 150-160

23. Manor E, Schmitz E, Sarov I. TNF $\alpha$, IL-1 and PGE production by human monocyte-derived macrophages infected with C. trachomatis. In: Bowie WR, Caldwell HD, Jones RP et al. (eds) Chlamydial infections. Proceedings of the 7th International Symposium on human chlamydial infections, Harrison Hot Springs. Cambridge, Cambridge University Press. 1990: 209-212. 
24. Tamura A, Matsumoto A, Manire GP, Higashi N. Electron microscopic observations on the structure of the envelopes of mature elementary bodies and developmental reticulate forms of Chlamydia psittaci. J Bacteriol 1971; 105: 355-360.

25. Newhall WJ. Macromolecular and antigenic composition of chlamydiae. In: Barron A (ed). Microbiology of Chlamydia. Boca Raton, Florida, CRC Press. 1988: 47-69.

26. Zvillich M, Sarov I. The persistence of Chlamydia trachomatis elementary body cell walls in human polymorphonuclear leucocytes and induction of a chemiluminescent response. $J$ Gen Microbiol 1989; 135 : 95-104.

27. Spedding G. Isolation and analysis of ribosomes from prokaryotes, eukaryotes, and organelles. In: Spedding G (ed) Ribosomes and protein synthesis: a practical approach. Oxford, IRL Press. 1990: 1-9.

28. Rahman MU, Cheema MA, Schumacher HR, Hudson AP. Detection of chlamydial RNA in the synovium of Reiter's syndrome patients by molecular hybridization. Arthritis Rheum 1990; 33 Suppl: 25 (abstract). 